\section{Integrative Medicine \\ International}

\title{
Elevated Hemidiaphragm and Shortness of Breath: Challenges in Clinicoradiologic Integration
}

\author{
Vivek Podder ${ }^{a, b} \quad$ Rakesh Biswas $^{b} \quad$ Nidhi Sehgal $^{c}$ \\ a Q City Medical College and Narayana Multispecialty Hospital, Durgapur, India; \\ ${ }^{b}$ Department of Medicine, Kamineni Institute of Medical Sciences, Narketpally, India; \\ 'Department of Radiology, IQ City Medical College and Narayana Multispecialty \\ Hospital, Durgapur, India
}

\section{Keywords}

Diaphragmatic paralysis · Chilaiditi's sign · Stable angina $\cdot$ Shortness of breath

\begin{abstract}
Unilateral diaphragmatic paralysis is often suspected when a hemidiaphragm is found abnormally elevated on chest radiography. Chest radiography has a high sensitivity for diaphragmatic palsy. A 67-year-old male presented to the hospital with a history of 4 months of exertional breathlessness that improved after taking a rest. The patient had normal cardiac and respiratory physical function. He also had a normal electrocardiogram and good systolic function on echocardiography. A treadmill test revealed ST-segment changes during peak exercise and recovery phases. On chest X-ray, his right hemidiaphragm was shown to be significantly elevated. Further investigation was conducted to rule out diaphragmatic paralysis, and the findings took a surprising turn to an entirely different diagnosis. (c) 2018 The Author(s)

Published by S. Karger AG, Basel
\end{abstract}

\section{Case Report}

A 67-year-old male presented to our outpatient department complaining of shortness of breath while walking upstairs that improved after taking a rest. He had noticed this for the last 4 months. He had been found to have hypertension for 4 years and had a transurethral resection of his prostate for lower urinary tract symptoms due to benign prostatic hyper-

Vivek Podder is a BMJ Case Reports Elective (https://promotions.bmj.com/jnl/bmj-case-reports-studentelectives-2/). 


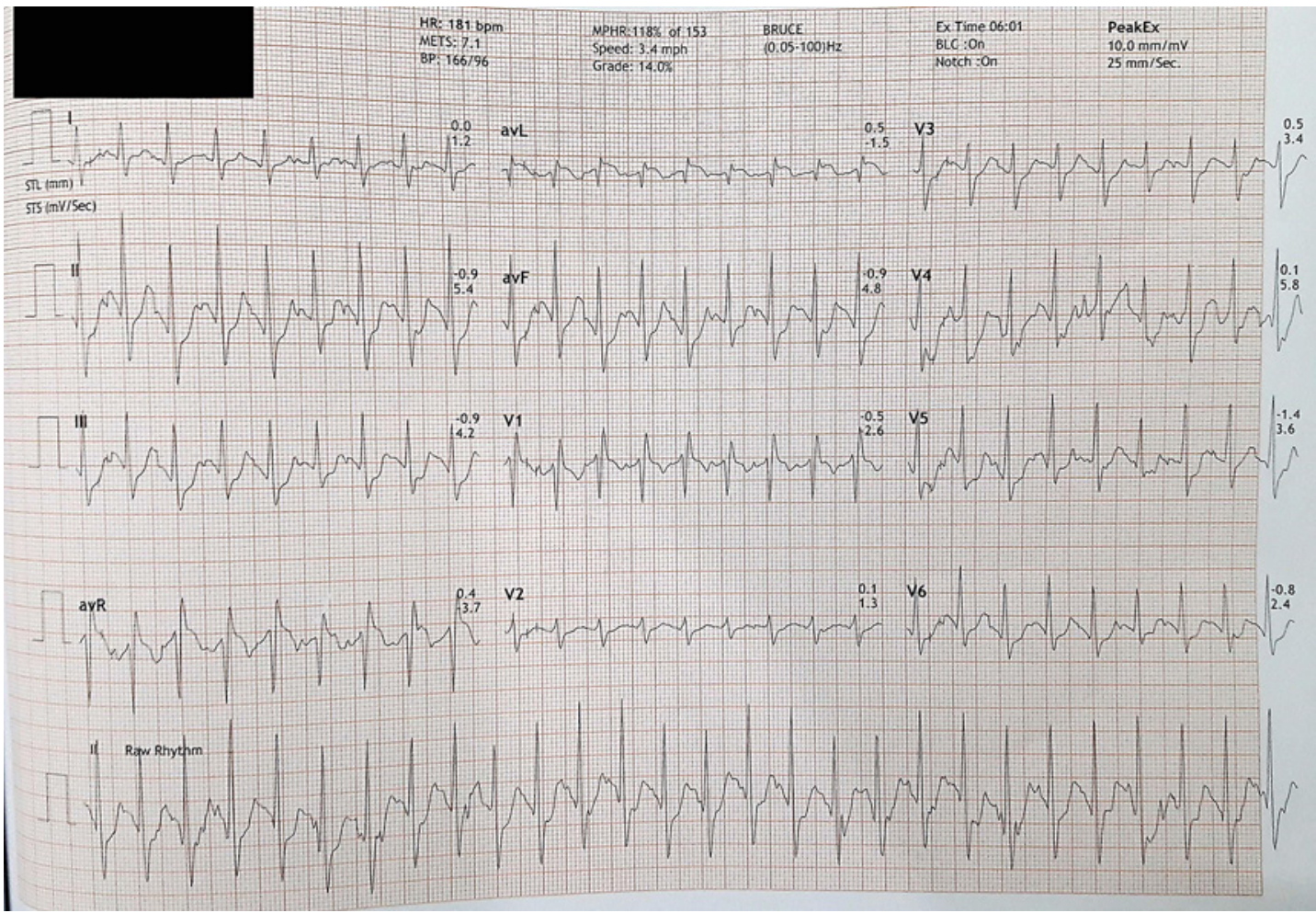

Fig. 1. ST-segment changes seen in the aVL lead during peak exercise in the treadmill test.

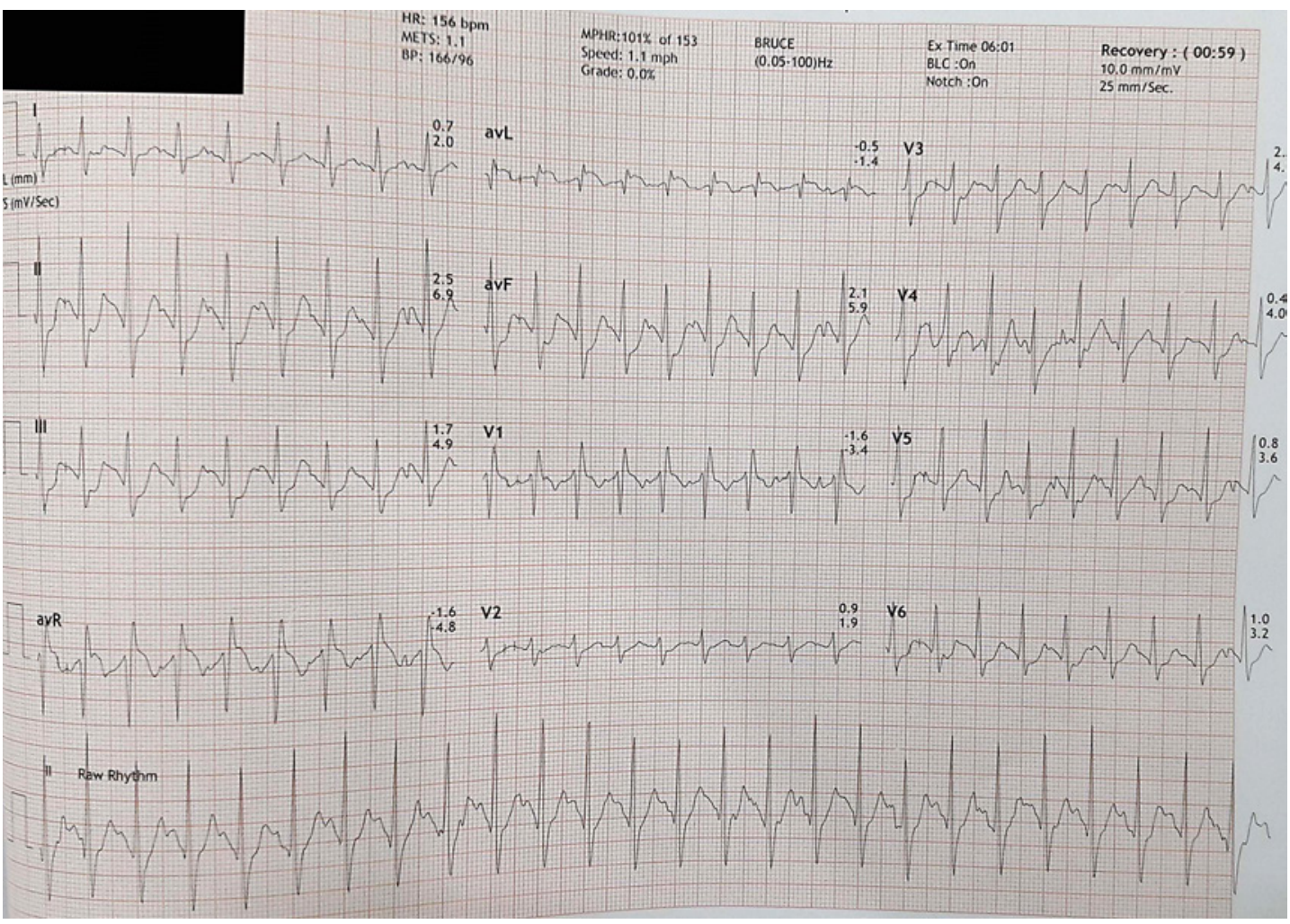

Fig. 2. ST-segment changes seen in the aVL lead during the recovery phase during the treadmill test. 
Fig. 3. Elevation of both the right and the left dome of the diaphragm seen on posteroanterior view of the chest X-ray.

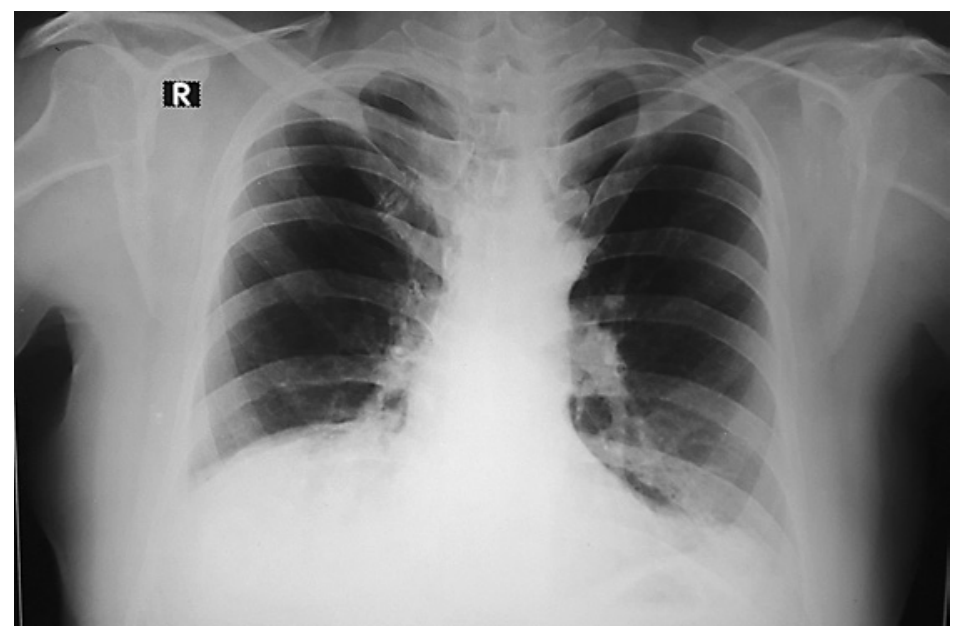

plasia. He had also been operated in the past for a left and right inguinal hernia surgery and had undergone a tonsillectomy at the age of 12 years. He has never smoked in his life. On examination, he had a normal first and second heart sound and normal breath sounds. His jugular venous pressure was normal. He had truncal obesity and gynecomastia.

This patient presented to us after some examinations by the previous facility, such as urinary routine microscopic examination to screen for any urinary tract infection in the background of his benign prostatic hyperplasia. The results were within the normal range, except for mild microalbuminuria, which reflected an early renal injury due to his hypertension. An electrocardiogram showed a sinus rhythm and echocardiography showed normal left ventricular systolic function with an ejection fraction of $62 \%$.

Further investigation by treadmill test showed ST-segment changes in the aVL lead during peak exercise (Fig. 1) and the recovery phase (Fig. 2). On a chest radiogram (posteroanterior view), we noticed a significant elevation in the right dome of his diaphragm (Fig. 3). Unilateral diaphragmatic palsy is often associated with the finding of an elevated hemidiaphragm on chest radiography, which is defined as the right dome of the diaphragm sitting $>2 \mathrm{~cm}$ higher than its left counterpart or the left hemidiaphragm sitting equally high or higher than its right counterpart. Chest radiography has a high sensitivity for diaphragmatic palsy but has a low positive predictive value. Diaphragmatic elevation has some common alternative causes such as Morgagni hernia, hiatal hernia, and a pulmonary or mediastinal mass and subphrenic abscess [1].

At this juncture, we wanted to rule these alternatives out, also to see if there was some association between his symptoms and possible diaphragmatic palsy. We evaluated the patient first by ultrasonography (USG) of the abdomen, which showed no paradoxical movement in the diaphragm but was unable to show any clear structures under the right dome of the diaphragm due to it being full of gas.

This was a point where we needed another imaging modality to resolve our diagnostic uncertainty, and a computed tomography (CT) scan was done. The CT scan of the abdomen revealed gas-filled colonic loops between the right hemidiaphragm and the liver (Fig. 4, 5).

The first apparent diaphragmatic palsy with shortness of breath took a surprising turn to an entirely different diagnosis. The colonic loops behind and above the liver and below the right dome of the diaphragm were interpreted as Chilaiditi's sign, which, needless to say, was incidental, but the actual cause of the patient's shortness of breath on unaccustomed exercise was defined by the treadmill test that showed significant changes at peak exercise and recovery. 
Integrative

Medicine

International

\begin{tabular}{l|l}
\hline Integr Med Int 2017;4:223-229 \\
\hline DOI: 10.1159/000494618 & $\begin{array}{l}\text { @ 2018 The Author(s). Published by S. Karger AG, Basel } \\
\text { www.karger.com/imi }\end{array}$ \\
\hline
\end{tabular}

Podder et al.: Elevated Hemidiaphragm and Shortness of Breath

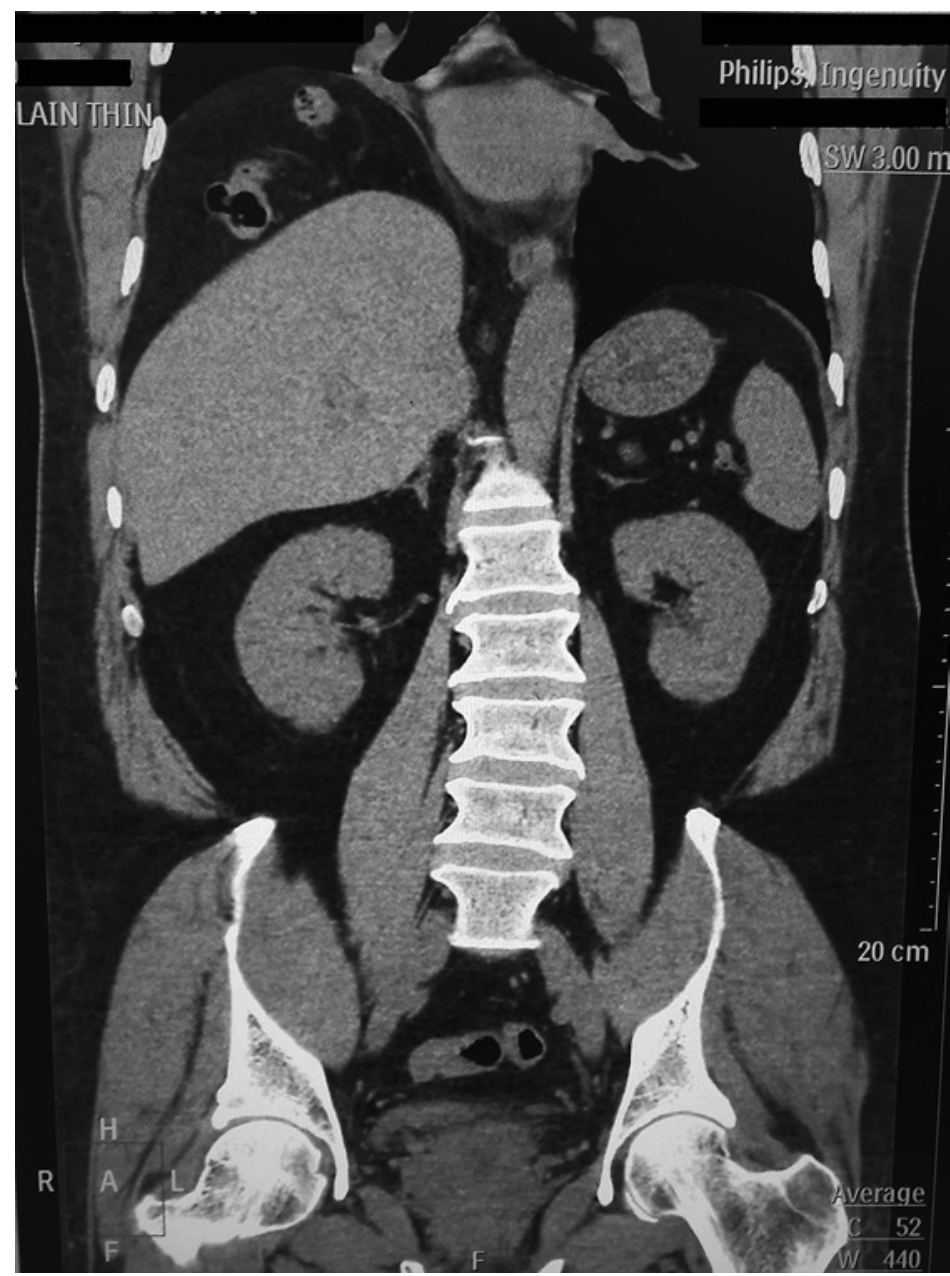

Fig. 4. Gas-filled colonic loops between the liver and the right dome of the diaphragm as seen on the computed tomography scan of the abdomen. 
Integrative

international

Fig. 5. Gas-filled colonic loops behind and above the liver and below the right dome of the diaphragm as seen on the computed tomography scan of the abdomen.

\begin{tabular}{l|l}
\hline Integr Med Int 2017;4:223-229 \\
\hline DOI: 10.1159/000494618 & $\begin{array}{l}\text { ○ 2018 The Author(s). Published by S. Karger AG, Basel } \\
\text { www.karger.com/imi }\end{array}$ \\
\hline
\end{tabular}

Podder et al.: Elevated Hemidiaphragm and Shortness of Breath

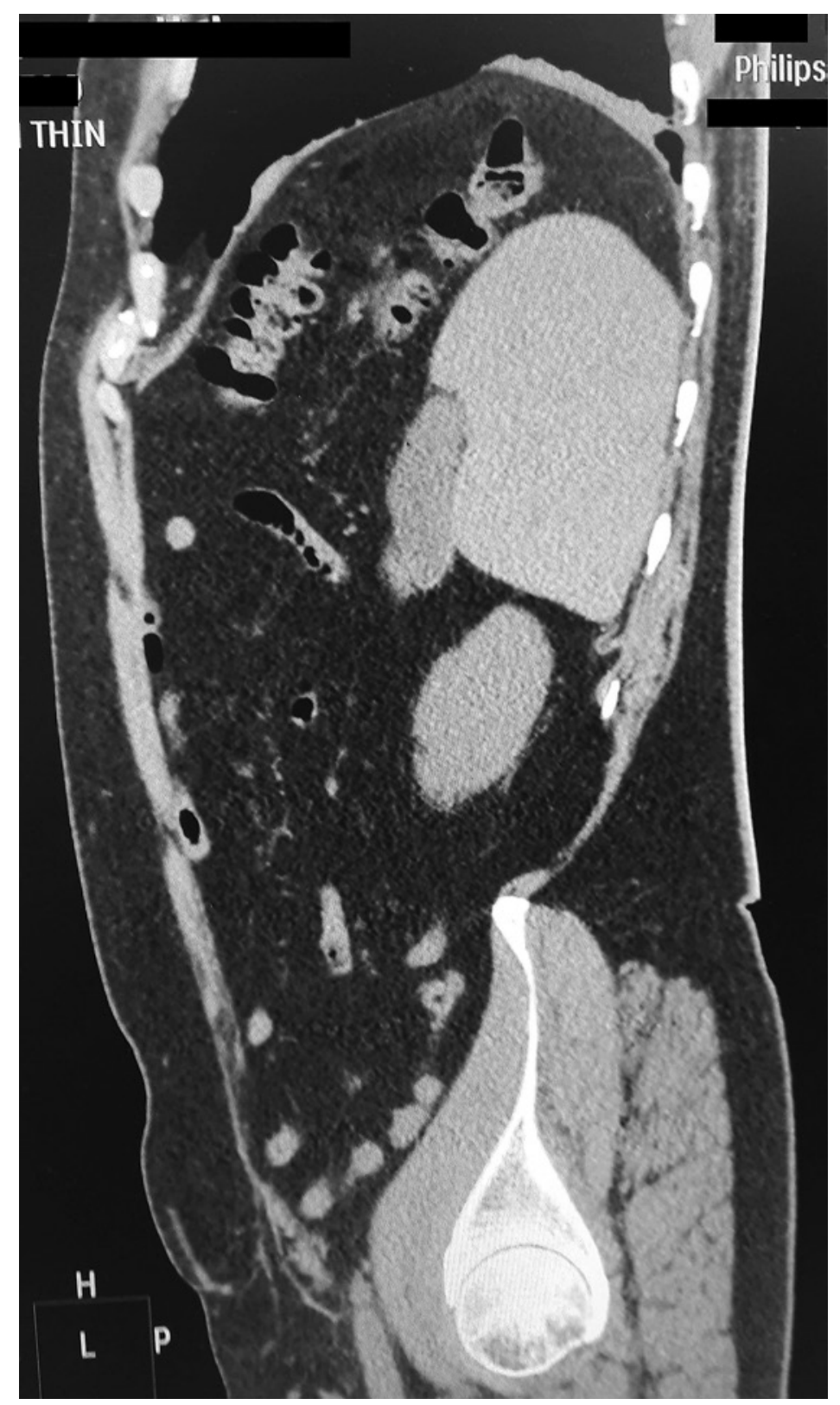

reported in a small case series on 3 patients who had the colon interposed between the liver and the right hemidiaphragm. Most patients with this condition remain asymptomatic throughout their life, and the condition is often incidentally detected on chest radiography or CT scans of the abdomen. This radiographic finding must be distinguished from Chilaiditi's syndrome, which is defined by the presence of clinical symptoms associated with the hepatodiaphragmatic interposition of the colon [2,3]. The symptoms most commonly manifest in patients with Chilaiditi's syndrome are gastrointestinal (e.g., nausea, vomiting, abdominal pain, distension, and constipation), respiratory (e.g., dyspnea and distress), and occasionally angina-like chest pain. Chilaiditi's syndrome is more often observed among older people, and males are 4 times more frequently affected than females. Conditions that increase the right subdiaphragmatic space and intestinal hypermobility predispose patients to develop this colonic interposition. Several predisposing factors have been implicated in the development 
of Chilaiditi's sign, broadly categorized into diaphragmatic, intestinal, hepatic, and other causes including abdominal obesity. Patients with liver cirrhosis, chronic obstructive pulmonary disease, or mental retardation have an increased prevalence of this condition. In the current patient, central obesity may have predisposed him to develop this interposition [4].

Pneumoperitoneum and subphrenic abscess are two important differential diagnoses of this radiographic sign. Other differential diagnoses include bowel obstruction, volvulus, and diaphragmatic hernia [3]. Historically, Chilaiditi's sign, also known as pseudopneumoperitonium, had been misinterpreted on chest X-ray as true pneumoperitoneum (free air in the peritoneal cavity). Physicians should carefully investigate to rule out this serious condition, as this may lead to unnecessary surgeries. Gas-filled colonic haustrations or normal plicae circulares under the right diaphragm can help distinguish Chilaiditi's sign from true pneumoperitoneum [4]. Chilaiditi's sign is diagnosed based upon findings observed on plain radiograms and CT scans. The associated radiography findings are characterized by elevation of the right dome of the diaphragm above the liver by the intestine, distension of the bowel by air, and depression of the superior margin of the liver below the level of the left dome of the diaphragm. For patients with liver cirrhosis, recognition of this sign is mandatory before any percutaneous transhepatic procedure such as liver biopsy is undertaken, to prevent intestinal injury. CT scanning is recommended to establish an accurate diagnosis if it cannot be achieved by radiography or ultrasound [3]. In this case, the right diaphragmatic elevation was incidentally detected on chest X-ray without any evidence of colonic gas shadows under it. The initial suspicion of diaphragmatic palsy turned into a different diagnosis on abdominal CT. This finding makes an important point, namely, that Chilaiditi's sign need not always have a visible colonic gas shadow on chest X-ray, as that may be detected on abdominal CT.

\section{Conclusions}

Diaphragmatic palsy can be a differential diagnosis for an elevated right dome of the diaphragm, but Chilaiditi's sign should also be considered if USG shows no findings positive for diaphragmatic palsy. Chilaiditi's sign need not always have visible gas shadows on chest $\mathrm{X}$-ray, as that may be detected on abdominal CT. Shortness of breath can be due to stable angina, which may not be attributed to the presence of Chilaiditi's sign.

\section{Acknowledgements}

We acknowledge Dr. Bhavik Shah for his initial review and editing of our manuscript.

\section{Statement of Ethics}

The study subject has given written informed consent to publish the details and photographs of the case.

\section{Disclosure Statement}

The authors have read and understood the Integrative Medicine International policy on declarations of interest, and the authors declare no conflicts of interest. 


\section{Availability of the Data}

Any further requests for information can be made by contacting R.B., the corresponding author.

\section{References}

1 Dubé BP, Dres M. Diaphragm Dysfunction: Diagnostic Approaches and Management Strategies. J Clin Med. 2016 Dec;5(12):E113.

2 Saber AA, Boros MJ. Chilaiditi's syndrome: what should every surgeon know? Am Surg. 2005 Mar; 71(3): 261-3.

3 Moaven O, Hodin RA. Chilaiditi syndrome: a rare entity with important differential diagnoses. Gastroenterol Hepatol (N Y). 2012 Apr;8(4):276-8.

4 Yin AX, Park GH, Garnett GM, Balfour JF. Chilaiditi syndrome precipitated by colonoscopy: a case report and review of the literature. Hawaii J Med Public Health. 2012 Jun;71(6):158-62. 